\title{
A Review of Ethics in Health Services and Policy: A Global Approach
}

\author{
Emma McKim Mitchell, PhD RN \\ Assistant Professor at the University of Miami \\ School of Nursing and Health Studies
}

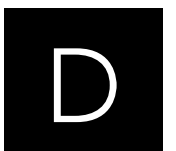

ean M. Harris, JD, MPH, is Clinical Associate Professor, Health Policy and Management, at the Gillings School of Global Public Health, University of North Carolina at Chapel Hill. He teaches healthcare law and globalization in healthcare at the undergraduate, Master's and doctoral level. His 2011 text explores ethical issues in healthcare policy and practice from a global perspective. Noted by the author in the introduction: the content has particular relevance for students in the healthcare professions - public health, medicine, nursing, and allied health. Two central themes of the book emerge: (1) exploring whether theories of ethics are globally applicable, and (2) exploring which theories help people decide how to "do the right thing" (p. 3). In other words, as Harris succinctly states: "are these ethical theories really global ... [and are they] really useful?" (p. 6).

The book begins with a discussion of the fundamentals of existing ethical theories. Each chapter starts by clarifying terms and introductory remarks about the purpose and content of each topic. Throughout the text are examples of situations where these theories may be applied globally, with the intention of challenging readers to critically analyze the applicability of such theories in the context of different cultures. In addition to examples embedded in each section, chapters all conclude with a critical-thinking exercise or activity. Also included in many chapters are excerpts from experts on relevant topics, or excerpts of seminal works on global health ethics. The author notes that after the reader has read the initial chapter outlining fundamental ethical theories, all chapters and content can be interchanged or read non-sequentially. In such a format, content can easily be incorporated into existing courses or coursework for healthcare professionals.

Harris explores a wide range of ethical issues, categorized here as (1) research issues such as autonomy and informed consent as they apply to global research with human subjects, (2) practice issues, including the right to healthcare and the healthcare practitioner's obligation to provide care, as well as the cultural ramifications of physician-assisted suicide and withholding/withdrawing treatment, (3) ethical issues in reproductive health, e.g., abortion and emergency contraception, as well as female genital mutilation, (4) healthcare service delivery issues including rationing and implications of allocating limited resources, issues of health insurance and health system reform, and corruption 
and informal payments in health systems, and (5) healthcare workforce issues, including movement of patients and healthcare practitioners across national borders.

Harris employs a comparative, multicultural model throughout the text. Within each chapter Harris poses several questions, encouraging readers to question preconceived ideas about "other" or "different" cultures and value systems. Such continued self-reflection is crucial for healthcare providers to ensure culturally competent and culturally inclusive care. For example, when discussing reproductive health, Harris acknowledges the deep-seated emotions and "gut reactions" groups often feel about issues such as abortion and emergency contraception. Through posing clarifying and interrogative questions, Harris challenges readers to examine the reproductive ethical issues prescribed by their own culture, as well as consider the perspectives of others'. Harris also discusses the historical progression of ethical issues in global healthcare, from individual patient/practitionercentred foci to contemporary discourse on social justice and the health of populations.

In addition to principles and exploring global health paradigms, two specific ethical dilemmas Harris discusses in more depth are physician-assisted suicide and female genital mutilation. Each is used to highlight the inextricable connection of ethical principles and the cultural context within which people experience health. Harris discusses economic considerations as well in the ethics of withdrawing or withholding treatment and of physician-assisted suicide.

In the eloquent excerpt from Marshall and Koenig (2004) included in chapter 1, Harris quotes: "We are deeply concerned with the implications of exporting American bioethics practices throughout the world. The problem is not simply one of national wealth or access to resources, although these are critical considerations" (Marshall and Koenig 2004: 9). Critical thinking exercises and case study vignettes throughout on global healthcare ethical principles help students analyze not only the research ethics model used within the United States, but also how this model can or cannot - and perhaps more aptly should or should not - apply in research and practice settings globally. Through exploring issues of autonomy and informed consent, and then encountering specific ethical dilemmas, students are presented with tools necessary for considering all angles of these complex situations.

One question I was left with at the end of the text, since it is directed at students in healthcare fields, concerns the ethical issues surrounding student global health service trips or practica. Perhaps within the chapter on healthcare workforce migration, discussion of the ethical issues of global health in student learning and practical experiences could have helped students not only consider broad ethical issues, but also individual practice and professional issues. Most often, healthcarerelated and academically affiliated service or service-learning trips state as a goal to increase access to healthcare resources during their 2-to-3 week immersion trips in "resource-poor" settings. They often lack evaluative mechanisms, or open dialogue about the ethical issues of transplanting (or "exporting" per Marshall and Koenig 2004) practice from one culture to another. Though not only a student phenomenon or an academically affiliated group phenomenon, this ethical issue warrants further discussion and exploration.

Regardless of the one question noted above, this comprehensive text is an excellent reference of ethical issues in global health practice and policy for healthcare professional students. Reading this text will challenge these students to explore their own ideas about ethics and culture, which will ultimately help them develop core competencies in delivering or better understanding culturally competent healthcare.

\section{References}

Harris, D.M. 2011. Ethics in Health Services and Policy: A Global Approach. San Francisco, CA: Jossey-Bass.

Marshall, P. and B. Koenig. 2004. "Accounting for Culture in a Globalized Bioethics." Journal of Law, Medicine and Ethics 32(2): 252-6. 